\title{
DESCRIÇÃO DOS PROCEDIMENTOS DE CINTILOGRAFIA PULMONAR EM SERES HUMANOS E EM ANIMAIS DOMÉSTICOS: REVISÃO DE LITERATURA
}

Jéssica Leite Fogaça ${ }^{1}$, Michel de Campos Vettorato ${ }^{1}$, Ariane Dantas ${ }^{1}$, Vânia Maria Vasconcelos Machado $^{2}$, Marco Antônio Rodrigues Fernandes ${ }^{3}$; Aline Sousa Camargos ${ }^{4}$

${ }^{1}$ Aluno de Pós-gradução da Faculdade de Medicina Veterinária e Zootecnia - Campus de Botucatu (UNESP), Distrito de Rubião Junior. ${ }^{2}$ Docente da Faculdade de Medicina Veterinária e Zootecnia - Campus de Botucatu (UNESP), Distrito de Rubião Junior. ${ }^{3}$ Docente do Instituto de Biociência - Campus de Botucatu (UNESP), Distrito de Rubião Junior. ${ }^{4}$ Docente do Instituto Federal Goiano - Campus Morrinhos - Zona Rural, Morrinhos - GO. Email: Aline.camargos@ifgoiano.edu.br

\section{RESUMO}

A medicina nuclear é uma prática médica utilizada para o diagnóstico ou tratamento de algumas doenças. A cintilografia pulmonar é um dos exames de medicina nuclear mais frequentes em seres humanos e em animais domésticos (cães, gatos e cavalos). Devido as suas diferenças, esse trabalho propôs descrever a realização da cintilografia pulmonar na medicina humana e na veterinária por meio da literatura e apresentar as principais diferenças nos procedimentos realizados em cada espécie. Para a elaboração desta revisão foram utilizados livros, periódicos, sites com publicações online encontradas no Google Acadêmico, Scielo, Bireme e Pubmed. Por meio das descrições da cintilografia pulmonar apresentadas nessa revisão, foi possível identificar as principais diferenças entre as espécies analisadas, tais como, a utilização da dose, o uso de contenção química, as etapas do exame, o isolamento e as adaptações presente nos equipamentos utilizados.

Palavras - chave: Medicina Nuclear. Cintilografia Pulmonar. Radiofármacos.

\section{DESCRIPTION OF PULMONARY SCINTIGRAPHY PROCEDURES IN HUMANS AND DOMESTIC ANIMALS: LITERATURE REVIEW}

\begin{abstract}
Nuclear medicine is a medical practice used to diagnose or treat some diseases. Pulmonary scintigraphy is one of the most common nuclear medicine exams in humans and domestic animals (dogs, cats and horses). Due to their differences, this work proposed to describe the performance of lung scintigraphy in human and veterinary medicine through the literature and present the main differences in the procedures performed in each species. For the preparation of this review were used books, periodicals, sites with online publications found in Google Scholar, Scielo, Bireme and Pubmed. Through the descriptions of pulmonary scintigraphy presented in this review, it was possible to identify the main differences among the analyzed species, such as dose utilization, use of chemical containment, the stages of the examination, isolation and adaptations present in the equipment used.
\end{abstract}

Keywords: Nuclear Medicine. Pulmonar Scintigraphy. Radiopharmaceuticals. 


\section{INTRODUÇÃO}

A medicina nuclear é uma prática médica utilizada para o diagnóstico ou tratamento de algumas doenças. Essa prática utiliza radionuclídeos, denominados de isótopos ou traçadores, aos quais são ligados a um composto químico (fármaco), formando assim o radiofármaco (radionuclídeo + fármaco). O radiofármaco é administrado no paciente por via intravenosa, oral ou inalatória. Após a realização deste procedimento, o paciente se torna uma fonte emissora de radiação ionizante (DIAS, 2009; NETO, 2012).

A cintilografia é uma técnica de medicina nuclear que oferece uma abordagem simples e não invasiva para investigar processos biológicos in vivo (STEYN, 1997; NETO, 2012; HUAIJANTUG, 2015). Esse método na medicina humana como na medicina veterinária tem mostrando crescente popularidade para pesquisas acadêmicas e para exames clínicos, devido a sua eficácia (WALMSLEY, 1995; HUAIJANTUG, 2015).

Uma das vantagens da cintilografia é possibilidade de visualizar e quantificar a distribuição de diferentes radiofármacos no organismo in vivo. A radiação gama proveniente do organismo do paciente é detectada por um equipamento específico, denominado de gamacâmara. Esse aparelho é responsável por converter a radiação gama em imagens cintilográficas (DUARTE et al., 2003; SANTOS et al., 2006; BARRAL et al., 2012).

A gama-câmara foi desenvolvida para detecção de tomografia computadorizada de fóton único (SPECT) e tomografia por emissão de pósitron (PET), proporcionando também imagens estáticas ou planares bidimensional (BALOGH et al., 1999; NETO, 2012).

A maioria das técnicas com os radionuclídeos utilizados em humanos (BARBIERI et al., 1984; MORAES et al., 2010; VIEIRA et al., 2011), também são aplicadas em pequenos e grandes animais (ARCHER et al., 2003; KRZEMIŃSKI et al., 2004; RODRIGUES et al., 2009; DANIEL; NEELIS, 2014). Porém, o que diferencia, são alguns procedimentos praticados no setor de medicina nuclear veterinária (KRZEMINSKI et al., 2004). A cintilografia pulmonar é um dos exames mais frequentes na medicina humana e na veterinária. Devido as suas diferenças, esse trabalho propôs descrever a realização da cintilografia pulmonar na medicina humana e na veterinária por meio da literatura e apresentar as principais diferenças nos procedimentos realizados em cada espécie.

\section{DESENVOLVIMENTO}

Para a elaboração desta revisão foram utilizados livros, periódicos, sites com publicações online encontradas no Google Acadêmico, Scielo, Bireme e Pubmed. As informações obtidas sobre os procedimentos de cintilografia pulmonar foram separadas em categorias (peso, dose, radiofármaco, espécie, etapa e contenção química) e analisadas conjuntamente.

A cintilografia pulmonar em seres humanos é amplamente utilizada para o diagnóstico de embolia pulmonar. O exame cintilográfico é realizado em duas etapas, denominadas de ventilação e perfusão pulmonar (SILVA; MULLER, 2004; CARVALHO et al., 2005).

Os radiofármacos utilizados para a fase de ventilação pulmonar são classificados por meio de duas categorias, denominadas gases radioaerossóis e gases radioativos. Essa última, incluem o Xenônio-127 ( $\left.{ }^{127} \mathrm{Xe}\right)$, Xenônio-133 ( $\left.{ }^{33} \mathrm{Xe}\right)$ e o Criptônio-81m ( $\left({ }^{81 \mathrm{~m}_{\mathrm{Kr}}}\right)$, porém, o mais utilizado para formação das imagens de ventilação é o ${ }^{133}$ Xe. A desvantagem deste elemento é energia ser relativamente baixa e prejudicar a realização das imagens de ventilação, após o estudo de perfusão pulmonar com o tecnécio ( $99 \mathrm{~m}_{\mathrm{T}}$ ). Para evitar esse tipo de problema, recomenda-se a realização das imagens de ventilação com o ${ }^{133}$ Xe e após a de perfusão com o 99m Tc (BARBIERI et al., 1984; ATTENBURROW et al., 1989).

A dose aplicada ao adulto padrão de 70 quilo $(\mathrm{Kg})$ é de 10 a 20 milicurie (mCi) $(370$ a 740 
Megabecquerel ( $\mathrm{MBq})$ ). As imagens são adquiridas em três fases diferentes, denominadas de: inspiração única (fase wasb-in), fase de equilíbrio e fase de expiração ou clareamento (fase wasbout). As imagens de ventilação pulmonar requerem a colaboração do paciente para a sua elaboração. No caso, dos pacientes que possuem taquipnéia, o protocolo do exame não é alterado. Para realização da cintilografia, o paciente fica sentado, no entanto, o exame pode ser realizado em decúbito dorsal, conforme o estado físico. É importante relatar que a sequência das projeções seja a mesma para ventilação e perfusão pulmonar (BARBIERI et al., 1984; DINIZ, 2003; ZIESSMAN et al., 2015).

Um método considerado alternativo para os estudos de ventilação pulmonar realizados com os gases radioativos são os radioaerossóis. O radioaerossol mais utilizado é o

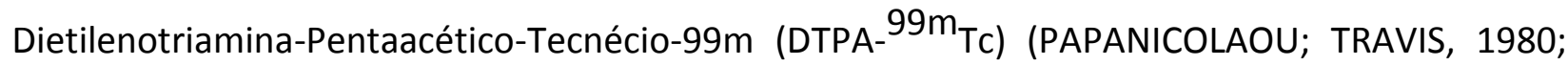
BARBIERI et al., 1984; PIVA et al., 2000; PAIVA et

al., 2014). A dose do radiofármaco selecionada para um adulto padrão de $70 \mathrm{Kg}$ é de 25 a $75 \mathrm{mCi}$ (925 a $1.875 \mathrm{MBq}$ ) de DTPA- ${ }^{99 \mathrm{~m}}$ Tc. As projeções das imagens realizadas são: anterior, posterior, lateral direita, esquerda, oblíquas posteriores direita e esquerda de 45 graus. O DTPA- ${ }^{99 m_{T c}}$ permanece no pulmão com uma meia vida biológica de 60 minutos, o que garante a produção de várias imagens cintilográficas (ZIESSMAN et al., 2015).

O diâmetro de uma hemácia é de aproximadamente 7,7 micrômetro $(\mu \mathrm{m})$. Caso partículas com diâmetro superior forem administradas via intravenosa, serão retidos nos précapilares do leito vascular do pulmão, capilares e arteríolas. Vários traçadores são constituídos de partículas biodegradáveis e não biodegradáveis e foram experimentalmente testados para estudos de cintilografia de perfusão pulmonar. Os traçadores mais utilizados para clínica médica são: Microesferas de Albumina Humana

Marcada com Tecnécio (MEAH- ${ }^{99} \mathrm{~m}_{\text {Tc }}$ ) e o Macroagregado de Albumina Marcada com Tecnécio (MAA- ${ }^{99 m}$ Tc). A dose usual de MAA- ${ }^{99 m}$ Tc para pacientes adultos padrão é de 1 a 5 mCi (40 a 150MBq). (BARRETO, 2004; KUBA, 2008; PAIVA et al., 2014). Após a realização do exame cintilográfico, o paciente é liberado no mesmo dia (MARTINS et al., 2001; MACEDO; RIYUZO; BASTOS, 2003; KUBA, 2008; BARBIRATO et al., 2009).

Os animais no setor de medicina nuclear são considerados como fonte emissora de radiação ionizante após aplicação do radiofármaco e por isso devem ser isolados em gaiola, baia ou tenda individual até que a radioatividade seja reduzida. Os resíduos sólidos encontrados na baia ou na gaiola que abriga o animal, durante 0 isolamento, ficam até 60 horas em um recipiente designado de chumbo (DANIEL, 2014).

Os animais variam relativamente de uma espécie para outra, sendo importante levar em consideração o tamanho, peso, características anatômicas e fisiológicas para realização dos exames. Os médicos veterinários baseiam os exames conforme realizado na medicina humana, mas existem algumas diferenças nos procedimentos (BALOGH et al., 2005) que devem ser relatados.

Os estudos de ventilação pulmonar têm sido predominantemente limitados a aplicação de pesquisas acadêmicas, devido a pouca colaboração do animal. Já, os estudos de perfusão pulmonar são mais realizados, devido à fácil contenção (BALOGH et al., 1999). A etapa de perfusão pulmonar é realizada com o mesmo radiofármaco da medicina humana. As doses dos radiofármacos variam conforme os métodos realizados, entre 0,54 a 4,0 mCi (20 a $150 \mathrm{MBq}$ ) cão e gato, e 15 a $20 \mathrm{mCi}$ (555 a $740 \mathrm{MBq}$ ) em equinos (BRAWNER; DANIEL, 1993; BALOGH et al., 1999) (RODRIGUES et al., 2009).

O tromboembolismo pulmonar, doença obstrutiva crônica e hemorragias são as clínicas mais comuns para cintilografia pulmonar em equinos. A distribuição dos radiofármacos nos 
pulmões é realizada em poucos minutos, no entanto, se houver ausência de perfusão de um segmento de pulmão, por qualquer motivo que seja, apresentará como pontos de pouca captação do radiofármaco. A cintilografia de ventilação pulmonar, também é um método que permite identificar estas alterações. Então é sempre recomendado fazer ambos os procedimentos (ventilação e perfusão), sempre que forem possíveis (BROM et al., 1989; BRAWNER; DANIEL, 1993).

A tabela a seguir, apresentada alguns dos principais procedimentos de cintilografia pulmonar descritos em seres humanos e em animais domésticos (cães, gatos e cavalos). Por meio da Tabela 1 é possível observar a metodologia utilizada em cada espécie e identificar as suas principais diferenças.

Tabela 1. Principais métodos utilizados no exame de cintilografia pulmonar em diferentes espécies.

\begin{tabular}{|c|c|c|c|c|c|c|}
\hline Referências & Espécie & Peso & $\begin{array}{c}\text { Procedimento } \\
\text { (Etana) }\end{array}$ & Radiofármaco & Dose & $\begin{array}{c}\text { Contenção } \\
\text { auímica }\end{array}$ \\
\hline $\begin{array}{l}\text { Papanicolao } \\
\text { u e Travis } \\
(1980\end{array}$ & $\begin{array}{l}\text { Humanos } \\
\text { (Pediátrico) }\end{array}$ & N.E. & $\begin{array}{c}\text { Ventilação/Perfus } \\
\text { ão }\end{array}$ & $\begin{array}{l}\text { DTPA- } \\
99 \mathrm{~m}_{\mathrm{Tc}} / \\
\text { MAA - }\end{array}$ & $\begin{array}{c}0,5-1 \mathrm{mCi} \\
(18,5-37 \\
\mathrm{MBq}) / \\
0,05\end{array}$ & N.E. \\
\hline $\begin{array}{l}\text { Alcoforado } \\
\text { et al. }\end{array}$ & $\begin{array}{l}\text { Humano } \\
\mathrm{s}\end{array}$ & N.E. & Ventilação & DTPA- ${ }^{99 m_{\mathrm{Tc}} /}$ & $\begin{array}{c}25 \mathrm{mCi} \\
(925\end{array}$ & Não \\
\hline $\begin{array}{l}\text { Brom et } \\
\text { al. }\end{array}$ & Cães & $\begin{array}{l}10-50 \\
\mathrm{Kg}\end{array}$ & $\begin{array}{c}\text { Ventilação/Perfus } \\
\text { ão }\end{array}$ & $\begin{array}{l}\text { Aerossol } \\
\text { (N.E.)/MAA }\end{array}$ & $\begin{array}{l}\text { N.E./ 1-2 } \\
\text { mCi (37- }\end{array}$ & $\begin{array}{c}\text { Sim } \\
\text { (Anestesia) }\end{array}$ \\
\hline $\begin{array}{c}\text { Clercx } \\
\text { of }\end{array}$ & Cães & $\begin{array}{c}8-18 \\
\mathrm{Ka}\end{array}$ & $\begin{array}{c}\text { Ventilação/Perfus } \\
\text { مصح }\end{array}$ & $\begin{array}{l}\text { DTPA- } \\
\text { a9m }\end{array}$ & N. E & $\operatorname{Sim}_{\text {Innactocial }}$ \\
\hline $\begin{array}{l}\text { Chetboul e } \\
\text { Delisle }\end{array}$ & Gato & $5,8 \mathrm{Kg}$ & Perfusão & MAA- ${ }^{99 m}$ Tc & $\begin{array}{c}250 \mathrm{mCi} \\
(9250\end{array}$ & $\begin{array}{c}\text { Sim } \\
\text { (Anestesia) }\end{array}$ \\
\hline $\begin{array}{l}\text { O'callagha } \\
\mathrm{n} \text { et al. } \\
(1987)\end{array}$ & Cavalos & N.E. & $\begin{array}{c}\text { Ventilação/Perfus } \\
\text { ão }\end{array}$ & $\begin{array}{l}\text { DTPA- } \\
99 \mathrm{~m}_{\mathrm{Tc}} / \\
\text { MAA - } \\
99 \mathrm{~m}\end{array}$ & $\begin{array}{c}4-5 \mathrm{mCi} \\
(148-185 \\
\mathrm{MBq}) / \\
20 \\
\end{array}$ & N.E. \\
\hline $\begin{array}{l}\text { Votion et } \\
\text { al. } \\
\text { (1997) }\end{array}$ & Cavalos & $493 \mathrm{Kg}$ & $\begin{array}{c}\text { Ventilação/Perfus } \\
\text { ão }\end{array}$ & $\begin{array}{l}\text { Nanocolóide } \\
\text { de } \\
\text { Albumina } \\
\text { Humana } \\
\text { / MAA- } \\
99 \mathrm{~m}_{\mathrm{Tc}}\end{array}$ & $\begin{array}{c}0,2 \\
\mathrm{mCi} / \mathrm{Kg} \\
(7,4 \\
\mathrm{MBq} / \mathrm{Kg}) \\
/ 0,03 \\
\mathrm{mCi} / \mathrm{Kg}\end{array}$ & $\begin{array}{c}\operatorname{Sim} \\
\text { (Sedação) }\end{array}$ \\
\hline
\end{tabular}

*N. E. Não Especificado 
Ao decorrer da revisão, notou-se que alguns autores Papanicolaou e Travis (1980), Alcoforado et al. (2011) e O'callaghan et al. (1987), não especificaram (N.E.) o peso e a contenção química dos animais utilizados nos exames. No entanto, outros autores Brom et al. (1989), Chetboul e Delisle (1997) e Votion et al. (1997) relataram o dose por peso dos animais e também descrevem o uso de contenção química.

A medicina veterinária realiza os exames baseados na medicina humana (CLERCX et al.,1989; BROM et al., 1989; RODRIGUES et al., 2009), mas como observado, não existe dose padrão para cada espécie (BALOGH et al., 2005).

Conforme ilustrado na Tabela 1, um dos trabalhos Chetboul e Delisle (1997) utilizou uma dose excessiva de $250 \mathrm{mCi}(9250 \mathrm{MBq}$ ) em um gato de 5,8 $\mathrm{Kg}$ na etapa de perfusão pulmonar. Essa dose se torna relativamente alta quando comparada com a de um cavalo Votion et al. (1997) e de um humano adulto Alcoforado et al. (2011).

Por meio da revisão, notou-se que a medicina humana prefere realizar as duas etapas da cintilografia pulmonar (ventilação e perfusão) (Papanicolaou e Travis (1980); Ziesmann et al., 2015). No entanto, pode ser realizado apenas uma etapa de exame, como foi do Alcoforado et al. (2011).

Com relação aos radiofármacos, foi observado que o DTPA- ${ }^{99} \mathrm{~m}_{\text {Tc e o MAA- }}$

$99 \mathrm{~m}$ Tc são os mais utilizados em humanos e animais. Já o ${ }^{133}$ Xe também pode ser aplicado para ambas espécies, mas o custo elevado e o tempo de meia vida curta minimiza a sua utilização. 0

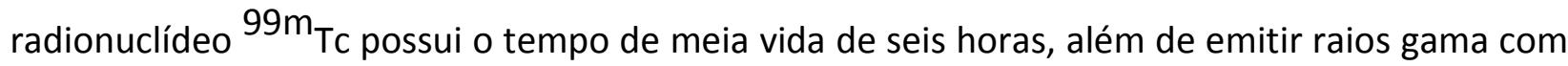
energia suficiente para a formação de imagens (ATTENBURROW et al., 1989).

O exame cintilográfico tanto na medicina humana como na medicina veterinária, dura em média de 20 a 30 minutos cada etapa, totalizando entre 40 a 60 minutos o exame completo (ATTENBURROW et al., 1989; ZIESSMAN et al., 2015). Os pacientes humanos não são submetidos a procedimentos anestésicos, a menos que seja sobre recomendação de médicos responsáveis pelo o setor. Já os pacientes veterinários na maioria dos exames os animais são sedados ou anestesiados.

O exame cintilográfico exige que o paciente permaneça imóvel sobre a mesa do equipamento gama-câmara. No entanto, a medicina humana como a veterinária utiliza softwares específicos para fazer o armazenamento e correção de artefatos de movimento da imagem. Entretanto os usos desses programas podem fazer com que a qualidade das imagens não seja tão fidedigna, então, recomenda-se sempre que possível iniciar o exame desde o começo (JARRETA, 2005; ZIESSMAN et al., 2015).

O equipamento gama-câmara utilizado na medicina veterinária geralmente são adaptáveis ao uso em grandes animais. Esse aparelho possui um apoio de rodas para a locomoção para fora da sala de exame, além de um controlador de altura para o detector, com ajustes conforme o local a ser examinado (ATTENBURROW, 1996).

Comparando os métodos realizados entre as espécies, observou-se que na medicina humana os pacientes são liberados no mesmo dia em que realizam os exames cintilográficos (MARTINS et al., 2001; MACEDO; RIYUZO; BASTOS, 2003; KUBA, 2008; BARBIRATO et al., 2009). Já, a medicina veterinária, opina em isolar os animais em gaiolas ou tendas individuais, durante horas ou dias, para garantir a segurança de todas as pessoas e animais em sua volta (MICHIELS et al., 2001; KAMPA et al., 2002; KRZEMIŃSKI et al., 2004; BALOGH et al., 2005; DANIEL et al., 2014).

\section{CONSIDERAÇÕES FINAIS}

Por meio das descrições da cintilografia pulmonar apresentadas nessa revisão, foi possível identificar as principais diferenças entre as espécies analisadas, tais como, a utilização 
da dose, o uso de contenção química, as etapas do exame, o isolamento e as adaptações presente nos equipamentos utilizados.

\section{REFERÊNCIAS}

ALCOFORADO, L. et al. Influência da variação dos decúbitos laterais na deposição pulmonar de aerossol. Brazilian Journal of Physical Therapy. v.15, n.4, p.278-283, 2011. https://doi.org/10.1590/S1413-35552011000400004.

ARCHER, D. C. et al. The normal scintigraphic appearance of the equine head. Equine Veterinary Education. v.15, n.5. p.243-249, 2003. http://dx.doi.org/10.1111/j.2042- 3292.2003.tb00535.x.

ATTEMBURROW, D. $P$ et al. Development of an equine nuclear medicine facility for gamma camera imaging. Equine Veterinary Journal. v.21, n.2, p.86-90, 1989. https://doi.org/10.1111/j.2042-3306.1989.tb02100.x.

ATTENBURROW, D. P. The application of scintigraphy using a gamma camera in clinical equine practice 1: Preparation and scanning procedure. Equine Veterinary Education. v.8, n.6, p.324328, 1996. http://dx.doi.org/10.1111/j.2042- 3292.1996.tb01715.x.

BALOGH, L. et al. Veterinary nuclear medicine again-commentary and remarks on: Krzeminski M et al. Veterinari nuclear medicine-a review. NMR 2004; 7: 177-182. Nuclear Medicine Review. Central \& Eastern Europe. v.8, n.1, p.50-52, 2005. Disponível em:

https://journals.viamedica.pl/nuclear_medicine_review/article/view/15327/12158.

Acesso em: 19 fev. 2017.

BALOGH, L. Veterinary nuclear medicine scintigraphical examinations - A Review. Journal Acta Veterinaria Brno. n. 68, p. 231-239, 1999. Disponível em file://C:/Users/Jessica/Downloads/Veterinary_nuclear_medicine_Scintigraphical_exami n\%20(2).pdf. Acesso em: 18 fev. 2017.

BARBIERI, A. et al. Radionuclídeos no exame do pulmão. Jornal de Peneumologia. v. 10, n.4, p.261-272, 1984. Disponível em https://books.google.com.br/books?id=DzMOs0ZSIr4C. Acesso em: 18 fev. 2017.

BARRAL, C. M. et al. The bone scintigraphy as a complementary exam in the diagnosis of the Avascular necrosis of the sesamoid. Brasileira Ortopédica. v. 47, n. 2, p. $241-245,2012$. Acesso em: 22 fev.2017. https://doi.org/10.1590/S0102-36162012000200016

BARRETO, S. S. M. Revisitando o mapeamento pulmonar de perfusão na tromboembolia pulmonar. Sociedade de Pneumologia e Tisiologia do Estado do Rio de Janeiro. v. 13, n. 4, p. 256-264, 2004. http://dx.doi.org/sopterj.com.br/profissionais/_revista/2004/n_04/07.pdf. Acesso em: 19 fev. 2017.

BRAWNER, W. R. DANIEL, G. B. Nuclear imaging. Veterinary Clinics of North America: Small Animal Practice. v. 23, n. 2, 20p, 1993. https://doi.org/10.1016/S0195-5616(93)50033-5

BROM, W. E. et al. Quantitative Analysis of Radioaerosol Inhalation and Perfusion Scintigraphy in Dogs. Lung Springer - Verlag New York. v. 167, p. 201-212, 1989. https://doi.org/10.1007/BF02714949. 
CARVALHO, M. R. M. et al. Embolia pulmonar no paciente idoso internado. Sociedade de Cardiologia do Rio de Janeiro. v.18, n.2, p.141-147, 2005. Disponível em: <http://www.rbconline.org.br/wp-content/uploads/a2005_v18_n02_art07.pdf.> Acesso em: 21 fev. 2017.

CHETBOUL, O. V. DELISLE, O. D. Diagnosis of pulmonary thromboembolism in a cat using echocardiography and pulmonary scintigraphy. Journal of Small Animal Practice. v.38, n.1, p.306-310, 1997. http://dx.doi.org/10.1111/j.1748- 5827.1997.tb03472.x.

CLERCX, C. et al. Pulmonary scintigraphy in canine lobarand sublobar airway obstruction. Lung Spinger Verlag. v.167, n.1, p.213-224, 1989. https://doi.org/10.1007/BF02714950.

DANIEL, G. B.; NEELIS, D. A. Thyroid scintigraphy in veterinary medicine. Sminars in nuclear medicine. v.44, n.1, p.24-34, 2014. https://doi.org/10.1053/i.semnuclmed.2013.08.007.

DANIEL. G. B. Guest Editoria: Veterinary nuclear medicine. Guest Editorial. v. 4, n. 1, 2p, 2014. https://doi.org/10.1053/i.semnuclmed.2013.08.001.

DIAS, A. L. Aplicação de nanopartículas magnéticas como meio de contraste computadorizada. 2009. 70f. Dissertação (Mestrado em Nanociências)-Universidade Francisco de Santa Maria, 2009. Disponível em http://tede.unifra.br/tde_arquivos/4/TDE-2009-07-07T071804Z39/Publico/Argleydson\%20Leao\%20Dias.pdf. Acesso em: 21 fev. 2017.

DINIZ, D. M. S. M. Padrão regional de ventilação pulmonar durante as técnicas do BreathStacking e inspirômetro de radioaerossol. 2003. 96f. Dissertação Mestrado (Departamento de Fisiologia e Farmacologia do Centro de Ciências Biológicas da Universidade Federal de Pernambuco, Recife, 2003. <http://repositorio.ufpe.br/handle/123456789/2154>. Acesso em: 21 fev. de 2017.

DUARTE, P. S. et al. O Significado clínico da dilatação transitória do ventrículo esquerdo avaliada pela cintilografia do miocárdio com ${ }^{99} \mathrm{~m}_{\mathrm{Tc}}$-sestamibi. Arquivo Brasileiro de Cardiologia. v.81, n.5, p.474-478, 2003. Disponível em <http://www.scielo.br/pdf/abc/v81n5/p04v81n5.pdf>. Acesso em: 21 fev. de 2017.

HUAYANTUG, S. Nuclear scintigraphic examination in veterinary medicine. Journal of Apllied Animal Science. v.8, n.1, p.9-16, 2015. Disponível em: http://www.vs.mahidol.ac.th/jaas/Files/Vol8No1/JAAS1(8)'15\%20RV\%20Somkiat.pdf. Acesso em: 23 fev. 2017.

JARRETA et al. Estudo do tempo de excreção renal pela cintilografia em felinos domésticos. Brazilian Journal of Veterinary Research and Animal Science. São Paulo, v.47, n.1, p.13-22, 2010. Disponível em: <http://producao.usp.br/handle/BDPI/1650>. Acesso: 23 fev. 2017.

FOGAÇA, J. L. Aplicações da cintilografia óssea em pequenos e grandes animais. Anual: V Jornada Científica e Tecnológica (Jornacitec), 2016. Disponível em: <http://www.fatecbt.edu.br/ocs/index.php/VJTC/VJTC/paper/view/805>. Acesso em: 24 de Fevereiro de 2016.

KRZEMINSKI, M. et al. Veterinary nuclear medicine. Nuclear Medicine Review. Central \& 
Eastern Europe. $\quad$ v.4, $\quad$ n.2, $2004 . \quad$ Disponível em: <https://journals.viamedica.pl/nuclear_medicine_review/article/view/15341/12172>. Acesso em: 23 fev. 2017.

KUBA. S. Instrumentação em medicina nuclear. 2008. 67p. Dissertação (Relatório Apresentado ao Instituto de Biociências de Botucatu) - Universidade Julio de Mesquita Filho - (Obtenção do Titulo de Bacharel em Ciências Biológicas) - Modalidade Médica. 2008. Disponível em: <http://repositorio.unesp.br/handle/11449/119549>. Acesso em: 23 fev. 2017.

MORAES et al. Cintilografia de perfusão miocárdica sob baixa dose de dobutamina na identificação do miocárdio viável. Radiologia Brasileira. v.43, n.5, 2010. https://doi.org/10.1590/S0100-39842010000500005.

NETO, J. A. S. Fator de correção de dose individual em grupos ocupacionais obtidos por monitoração individual externa em serviço de medicina nuclear. 2012, 65f. Dissertação (Pós Graduação em Tecnologias Energéticas e Nucleares do Departamento de Energia Nuclear) Universidade Federal de Pernambuco para Obtenção do Titulo de Mestre, 2012. Disponível em: <http://repositorio.ufpe.br/handle/123456789/10458>. Acesso em: 23 fev. 2017.

O' CALLAGHAN, M. W. et al. Exercise-induced pulmonary haemorrhage in the horses: results of a detailed clinical, post mortem and imaging study. VII Ventilation/perfusion scintigraphy in horses with EIPH. Equine Veterinary Journal. v. 19, n. 8, p. 423-427, 1987. Disponível em http://dx.doi.org/10.1053/j.semnuclmed.2013.08.00110.1111/j.2042- 306.1987.tb02636.x.

PAPANICOLAOU, N. TREVES, S. Pulmonary scintigraphy in pediatrics. Seminars in Nuclear Medicine. v.10, n. 3, p. 259-285. http://dx.doi.org/10.1016/S0001- 2998(80)80006-7.

PAIVA, D. N. et al. Efeito de diferentes níveis de pressão positiva continua sobre a $99 \mathrm{~m}$ depuração pulmonar do Tc-DTPA. Epidemiologia e Controle de Infecção. v.4, n.2, p.154-158, 2014. https://doi.org/10.17058/reci.v4i2.4646.

PIVA, J. et al. The use of helium-oxygen mixture in the ventilation study of children with chronic obstrutive lung disease. Jornal de Pediatria. v. 76, n. 1, p. 17-26, 2000. Disponível em: $<$ https://www.researchgate.net/profile/Flavio_Zelmanovitz/publication/8982846_The_us e_of_helium-

oxygen_mixture_in_the_ventilation_study_of_children_with_chronic_obstructive_lung_d isease/links/0deec522db87439dba000000.pdf>. Acesso em: 24 fev. 2017.

RODRIGUES, G. S. et al. Avaliação cintilográfica de diferentes dosagens de tecnécio na padronização da perfusão pulmonar em cães da raça Rottweller. Ciências Rural, Santa Maria. v.40, n.3,p.554-560, 2009. https://doi.org/10.1590/S0103-84782010005000022.

SANTOS, T. S. G. et al. Insuficiência cardíaca progressiva em homem de 44 anos de idade. Arquivo Brasileiro de Cardiologia. v. 86, n. 2, 2006. https://doi.org/10.1590/S0066782X2006000200013.

SILVA, C. I. MULLER, N. Diagnóstico por imagem do tromboembolismo pulmonar agudo. "Jornal Brasileiro de Pneumologia" v. 30, n. 5, p. 474-479, Set/Out 2004. Disponível em: <http://www.scielo.br/pdf/jbpneu/v30n5/v30n5a12>. Acesso em: 16 fev. 2017. 
STEYN, P. F. et al. The effect of intravenous drazepam on sold phase gastric emptyng in normal cats. Veterinary Radiology \& Ultrasound. v. 38, n. 6, p. 469-473, 1997. https://doi.org/10.1111/j.1740-8261.1997.tb00874.x.

VIEIRA, L. O. et al. Correlação entre volume tireoidiano determinado pelo método de ultrassonografia versus cintilografia e sua implicação em cálculos dosimétricos na terapia com radioiodo na doença de Graves. Arquivo Brasileiro Endocrinologia \& Metabólica. v. 55, n. 9, p. 5, Novembro, 2011 https://doi.org/10.1590/S0004-27302011000900005.

VOTION, D. et al. Analysis of Equine Scintigraphical Lung Images. The Veterinary Journal. v. 153, n. 1, p49-61, 1997. https://doi.org/10.1016/S1090-0233(97)80008-1.

WALMSLEY, J. P. Scintigraphy is here to stay. Equine Veterinary Education. v. 7, n. 3, p. $122-$ 123, 1995. https://doi.org/10.1111/i.2042-3292.1995.tb01206.x.

ZIESSMAN et al. Medicina Nuclear. 4 ed. Saunders Elsevier: Rio de Janeiro, 2015. 464p. 